Supporting Information for:

\title{
Topochemistry of Bowtie- and Star-Shaped Metal Dichalcogenide Nanoisland Formation
}

Vasilii I. Artyukhov*, Zhili Hu*, Zhuhua Zhang, and Boris I. Yakobson\#

Department of Materials Science and NanoEngineering, Rice University, Houston, TX 77005

\# corresponding author e-mail: biy@rice.edu

* these authors contributed equally

\section{Full simulation videos of polycrystal formation}

Movies S1 and S2 show, respectively, full evolution of simulated systems for Fig. 2f (bowtie) and Fig. 2 (star). In each movie, initial $t=10$ time units are first shown in slower time and zoomed-in to show island nucleation and collision events in detail, followed by full videos of the entire simulation domain to show the large-scale structure evolution.

\section{Phase field method details}

The multi-component phase-field (MPF) model developed by Steinbach and Pezzola33 served as the backbone in our simulations. Since the original MPF model does not consider the evolution of feedstock for epitaxial growth, here we advance a composite MPF model by augmenting it with the feedstock factor from a phase-field model by Karma and Rappel ${ }^{48}$ where the effects of adsorption and desorption are considered.

The multicomponent phase field model is specified as follows. It starts from a general free energy functional,

$$
G=\sum_{\alpha, \beta=1 \ldots N, \alpha>\beta} \frac{4 \sigma_{\alpha \beta}}{\pi^{2}}\left(-\eta \nabla \phi_{\alpha} \cdot \nabla \phi_{\beta}+\frac{\pi^{2}}{\eta} \phi_{\alpha} \phi_{\beta}\right)+\lambda \xi h\left(\phi_{N}\right),
$$

where $\sigma_{\alpha \beta}$ is either the grain-grain or grain-substrate interface energy between two phases $\alpha$ and $\beta$ (GB or edge tension, respectively); $\eta$ is the interface thickness, and $\varphi_{\alpha}$ is the order parameter of the $\alpha$-th phase, where $\alpha=1 \ldots(N-1)$ means $M X_{2}$ grain and $\alpha=N$ denotes the bare substrate, and we ensure that $\mathrm{o} \leq \varphi_{\alpha} \leq 1$. The interpolation function $h$ takes form of $\mathrm{h}(\varphi)=$ $2 / \pi(2 \varphi-1)[\varphi(1-\varphi)]^{1 / 2}+1 / \pi \arcsin (2 \varphi-1)$. The dimensionless supersaturation $\xi=\left(c_{N}-c_{\mathrm{eq}}\right) / c_{\mathrm{s}}$, where $c_{N}$ is the feedstock concentration, $c_{\text {eq }}$ is the equilibrium value of $c_{N}$, and $c_{\mathrm{s}}$ is the nominal concentration of precursor in solid 2D $M X_{2}$, provides the driving force to the grain growth with a coupling constant $\lambda$. For example, in the case of $\mathrm{MoS}_{2}$ grown via sulfurization of $\mathrm{MoO}_{3}$ on $\mathrm{SiO}_{2}, \xi$ corresponds to adsorbed $\mathrm{MoO}_{3}$, as it is the limiting step of the synthesis. ${ }^{6}$

In the simulation, each $M X_{2}$ phase $\varphi_{\alpha}$ is assigned with a specific grain orientation $\theta_{\alpha}$. The GB energy is then evaluated as $\sigma_{\alpha \beta}=\sigma_{1}\left|\sin \left(n \theta_{\alpha \beta}\right)\right|+\sigma_{2}\left|\sin \left(n /{ }_{2} \theta_{\alpha \beta}\right)\right|$ where $n=3$ so that $M X_{2}$ has a 3- 
fold symmetry, and $\theta_{\alpha \beta}=\left|\theta_{\alpha}-\theta_{\beta}\right|$ is the misorientation. With this formulation, $\sigma_{\alpha \beta}$ has a global minimum at $\theta_{\alpha \beta}=\mathrm{O}+2 \pi m / 3$, and a secondary local minimum at $\theta_{\alpha \beta}=\pi / 3+2 \pi m / 3$ with $m$ integer. The values of $\sigma_{1}$ and $\sigma_{2}$ are taken to fit the lower bounds of respective GB from DFT calculations 25. For grain edges, the interface tension is $\sigma_{a N}=\sigma_{0}\left[1+\delta \cos n\left(\psi_{\alpha}-\theta_{\alpha}\right)\right]$ where $\delta=0.1, \sigma_{0}=1$ $\mathrm{ev} / \AA$, and $\psi_{\alpha}$ is the orientation of the edge normal (gradient of $\varphi_{\alpha}$ ). An extra constraint on the order parameters is that they sum to unity, $\Sigma_{\alpha=1 \ldots N} \varphi_{\alpha}=1$.

The governing equation for order parameters satisfying the constraint is:33

$$
\dot{\phi}_{\alpha}=-\sum_{\beta=1 \ldots \widetilde{N}} \frac{2 \pi^{2}}{8 \eta} \cdot \frac{\mu_{\alpha \beta}}{\widetilde{N}}\left(\frac{\delta G}{\delta \phi_{\alpha}}-\frac{\delta G}{\delta \phi_{\beta}}\right)
$$

where $\widetilde{N}$ is the local number of coexisting phases, and $\mu_{\alpha \beta}$ is the interface mobility. For $\mathrm{MoS}_{2}$ GBs we set $\mu_{\alpha \beta}=\mu_{o}\left(\varphi_{N}\right)^{1 / 2}$ with $\mu_{0}$ a universal coefficient. This locks the shape of GB upon formation. At free edges the mobility is $\mu_{a N}=\mu_{0}\left[1+2.5\left|\cos n\left(\psi_{a}-\theta_{a}\right) / 2\right|\right]$. This form is chosen so that kinematic Wulff construction produces an equilateral triangular shape.

The detailed formulation for the evolution of order parameters is given in Ref. 49 and omitted here for brevity. The evolution of the supersaturation required to solve it is given by:48,50,51

$$
\xi=\nabla(D \nabla \xi)-\frac{\dot{\xi}}{\tau_{s}}+F+\frac{\partial h\left(\phi_{N}\right)}{\partial t}
$$

where $F$ is the deposition flux, $D$ is the diffusion coefficient of $\xi$, and $\tau_{s}$ is the desorption time. With constant $F$ and $\tau_{s}$ the supersaturation at equilibrium will be $\xi_{e q}=F \tau_{s}$. The diffusion coefficient $D$ is uniform across the substrate surface. Using units of characteristic length $l$, and time $\tau=l^{2} \widetilde{D} / D$, we can rewrite the equations with $\tilde{\tau}_{s}=\tau_{s} D /\left(l^{2} \widetilde{D}\right), \tilde{\mu}=\mu_{0} \widetilde{D} / D, \tilde{\sigma}=\sigma / \sigma_{1}$, and $\tilde{F}=\widetilde{D} l^{2} F / D$ as new parameters to obtain a dimensionless system. We solve it in Matlab by discretization with a time step of $0.01 \tau$ for the order parameters evolution, $0.0007 \tau$ for supersaturation evolution, and space grid resolution of $l$. The boundary conditions are periodic and fast Fourier transform is used as a highly effective of way to get field variable derivatives. The initial supersaturation is set to be at equilibrium everywhere, accordingly $F=\xi_{e q} / \tau_{s}$. Each nucleus starts from a circle with a radius of either $3 l$ or $5 l$.

Aside from GB energies based on literature DFT values, simulation parameters were determined empirically by tuning to reproduce the features of single-crystalline island growth. Experiment shows that if feedstock concentration is low, nearly perfect triangles grow, while 3-point-stars appear at high concentrations, ${ }^{7}$ which is consistent with onset of diffusion instability. Although the pressure of the feeding gas in experiments can differ by 5 magnitude of order under experimental conditions, computational limitations allow us only to explore the high supersaturation regimes. Thus the equilibrium concentration $\xi_{\text {eq }}$ was varied from 0.1 to 0.3 in the simulations. The coupling coefficient was held at a fixed value of $\lambda=20$ so that only $\xi_{\text {eq }}$ determines stability of growth shape. Other parameters are listed in Table S1. The results show that by varying $\xi_{e q}$ from low to high, the island shape evolves from a triangle (Fig. 2c) to a nonconvex 3-point star (Fig. 2d), as seen in experiments. ${ }^{6,7}$ 
Table S1. List of parameters for the phase-field simulation. Tildes are omitted for dimensionless parameters.

\begin{tabular}{lll}
\hline \hline Parameter & Symbol & Value \\
\hline GB energy $\left(C_{6}\right.$ term $)$ & $\sigma_{1}$ & $1 \mathrm{eV} / \AA$ \\
GB energy $\left(C_{3}\right.$ term $)$ & $\sigma_{2}$ & $0.5 \mathrm{eV} / \AA$ \\
Characteristic length & $l$ & $50 \mathrm{~nm}$ \\
Interface thickness & $\eta$ & $7 l$ \\
Coupling coefficient & $\lambda$ & 20 \\
Desorption time & $\tilde{\tau}_{s}$ & 2.5 \\
Diffusivity & $\widetilde{D}$ & 150 \\
Mobility & $\mu_{o}$ & 2 \\
\hline
\end{tabular}

\section{Analytical calculation of capillary torque}

The result that torque acting upon colliding islands scales as inverse square of the angle can be obtained in the harmonic approximations, assuming that the unit cells of edges are connected one-to-one with finite-range harmonic springs (bonds):

$$
\epsilon=\left\{\begin{array}{c}
\frac{k y^{2}}{2}, 0 \leq y<h \\
\frac{k h^{2}}{2}, y \geq h
\end{array}\right.
$$

Here, $y$ is the distance from a unit cell on one edge to its counterpart on the other and $h$ is the maximum (breaking) bond length. Integrating $\epsilon$ over all such pairs along the edge (as indexed by coordinate $x$ ), we get the total energy:

$$
E=\int_{0}^{h \cot (\alpha / 2)} \frac{k}{2}\left(2 x \sin \frac{\alpha}{2}\right)^{2} d x=\frac{2}{3} h^{3} k\left(\cos \frac{\alpha}{2}\right)^{3} / \sin \frac{\alpha}{2} \approx \frac{4 h^{3} k}{3 \alpha}
$$

A similar result is obtained for the Morse potential, $\epsilon=D\left(1-e^{-y \sqrt{k / 2 D}}\right)^{2}-D$, and integrating in $x$ to infinity:

$$
E=\int_{0}^{+\infty}\left(D\left(1-e^{-2 x \sin \frac{\alpha}{2} \sqrt{k / 2 D}}\right)^{2}-D\right) d x=\frac{3 \sqrt{D}}{2 \sqrt{2} k \sin \alpha / 2} \approx \frac{3 \sqrt{D}}{\sqrt{2} k \alpha}
$$

In both cases $E \propto 1 / \alpha$ and thus the torque $d E / d \alpha \propto 1 / \alpha^{2}$.

The above derivation assumed an infinitely stiff crystal lattice. For very small values of $\alpha$, local relaxation should create an upper bound on the torque determined by material elasticity. 


\section{References}

(1) Chhowalla, M.; Shin, H. S.; Eda, G.; Li, L.-J.; Loh, K. P.; Zhang, H. Nat. Chem. 2013, 5 (4), 263-275.

(2) Coleman, J. N.; Lotya, M.; O’Neill, A.; Bergin, S. D.; King, P. J.; Khan, U.; Young, K.; Gaucher, A.; De, S.; Smith, R. J.; Shvets, I. V.; Arora, S. K.; Stanton, G.; Kim, H.-Y.; Lee, K.; Kim, G. T.; Duesberg, G. S.; Hallam, T.; Boland, J. J.; Wang, J. J.; Donegan, J. F.; Grunlan, J. C.; Moriarty, G.; Shmeliov, A.; Nicholls, R. J.; Perkins, J. M.; Grieveson, E. M.; Theuwissen, K.; McComb, D. W.; Nellist, P. D.; Nicolosi, V. Science 2011, 331 (6017), 568-571.

(3) Lv, R.; Robinson, J. A.; Schaak, R. E.; Sun, D.; Sun, Y.; Mallouk, T. E.; Terrones, M. Acc. Chem. Res. 2015, 48 (1), 56-64.

(4) Lee, Y.-H.; Zhang, X.-Q.; Zhang, W.; Chang, M.-T.; Lin, C.-T.; Chang, K.-D.; Yu, Y.-C.; Wang, J. T.-W.; Chang, C.-S.; Li, L.-J.; Lin, T.-W.Adv. Mater. 2012, 24 (17), 2320-2325.

(5) Zhan, Y.; Liu, Z.; Najmaei, S.; Ajayan, P. M.; Lou, J. Small 2012, 8 (7), 966-971.

(6) van der Zande, A. M.; Huang, P. Y.; Chenet, D. A.; Berkelbach, T. C.; You, Y.; Lee, G.-H.; Heinz, T. F.; Reichman, D. R.; Muller, D. A.; Hone, J. C. Nat. Mater. 2013, 12, 554-561.

(7) Najmaei, S.; Liu, Z.; Zhou, W.; Zou, X.; Shi, G.; Lei, S.; Yakobson, B. I.; Idrobo, J.-C.; Ajayan, P. M.; Lou, J. Nat. Mater. 2013, 12 (8), 754-759.

(8) Zhang, Y.; Zhang, Y.; Ji, Q.; Ju, J.; Yuan, H.; Shi, J.; Gao, T.; Ma, D.; Liu, M.; Chen, Y.; Song, X.; Hwang, H. Y.; Cui, Y.; Liu, Z. ACS Nano 2013, 7 (10), 8963-8971.

(9) Liu, Z.; Amani, M.; Najmaei, S.; Xu, Q.; Zou, X.; Zhou, W.; Yu, T.; Qiu, C.; Birdwell, A. G.; Crowne, F. J.; Vajtai, R.; Yakobson, B. I.; Xia, Z.; Dubey, M.; Ajayan, P. M.; Lou, J. Nat. Commun. 2014, 5 .

(10) Kang, K.; Xie, S.; Huang, L.; Han, Y.; Huang, P. Y.; Mak, K. F.; Kim, C.-J.; Muller, D.; Park, J. Nature 2015, 520 (7549), 656-660.

(11) Artyukhov, V. I.; Liu, Y.; Yakobson, B. I. Proc. Natl. Acad. Sci. U. S. A. 2012, 109 (38), 15136-15140.

(12) Artyukhov, V. I.; Hao, Y.; Ruoff, R. S.; Yakobson, B. I. Phys. Rev. Lett. 2015, 114 (11), 115502.

(13) Liu, Y.; Bhowmick, S.; Yakobson, B. I. Nano Lett 2011, 11, 3113-3116.

(14) Cao, D.; Shen, T.; Liang, P.; Chen, X.; Shu, H. J. Phys. Chem. C 2015, 119 (8), 42944301.

(15) Ji, Q.; Kan, M.; Zhang, Y.; Guo, Y.; Ma, D.; Shi, J.; Sun, Q.; Chen, Q.; Zhang, Y.; Liu, Z. Nano Lett. 2015, 15 (1), 198-205.

(16) Cheng, J.; Jiang, T.; Ji, Q.; Zhang, Y.; Li, Z.; Shan, Y.; Zhang, Y.; Gong, X.; Liu, W.; Wu, S. Adv. Mater. 2015, 27 (27), 4069-4074.

(17) Lauritsen, J. V.; Kibsgaard, J.; Helveg, S.; Topsøe, H.; Clausen, B. S.; Lægsgaard, E.; Besenbacher, F. Nat. Nanotechnol. 2007, 2 (1), 53-58.

(18) Liu, B.; Fathi, M.; Chen, L.; Abbas, A.; Ma, Y.; Zhou, C. ACS Nano 2015.

(19) Gibbs, J. W. Trans. Conn. Acad. Arts Sci. 1876, 3 (5), 108-248.

(20) Wulff, G. Z. Für Kryst. Mineral. 1901, 34 (5/6), 449-530.

(21) Frank, F. C. In Growth and Perfection in Crystals; Doremus, R. H., Roberts, B. W., Turnbull, D., Eds.; John Wiley\& Sons Inc.: New York, 1958; p 411.

(22) Frank, F. C. Z. Für Phys. Chem. 1972, 77 (1-6), 84-92.

(23) Winterbottom, W. L. Acta Metall. 1967, 15 (2), 303-310.

(24) Ringe, E.; Van Duyne, R. P.; Marks, L. D. J. Phys. Chem. C 2013, 117 (31), 15859-15870.

(25) Zou, X.; Liu, Y.; Yakobson, B. I. Nano Lett. 2013, 13 (1), 253-258.

(26) Liu, Y.; Zou, X.; Yakobson, B. I. ACS Nano 2012, 6 (8), 7053-7058.

(27) Herring, C. Phys. Rev. 1951, 82 (1), 87-93.

(28) Herring, C. In Metal interfaces: a seminar on metal interfaces held during the Thirtythird National Metal Congress and Exposition, Detroit, October 13 to 19, 1951; 
sponsored by the American Society for Metals; American Society of Metals: Metals Park, Ohio, 1952; p 16.

(29) Zhang, K.; Hu, S.; Zhang, Y.; Zhang, T.; Zhou, X.; Sun, Y.; Li, T.-X.; Fan, H. J.; Shen, G.; Chen, X.; Dai, N. ACS Nano 2015, 9 (3), 2704-2710.

(30) Lehtinen, O.; Komsa, H.-P.; Pulkin, A.; Whitwick, M. B.; Chen, M.-W.; Lehnert, T.; Mohn, M. J.; Yazyev, O. V.; Kis, A.; Kaiser, U.; Krasheninnikov, A. V. ACS Nano 2o15, 9 (3), 3274-3283.

(31) Bets, K. V.; Artyukhov, V. I.; Yakobson, B. I. arXiv:1412.4323 2014.

(32) Fan, D.; Geng, C.; Chen, L.-Q. Acta Mater. 1997, 45 (3), 1115-1126.

(33) Steinbach, I.; Pezzolla, F. Phys. Nonlinear Phenom. 1999, 134 (4), 385-393.

(34) Azizi, A.; Eichfeld, S.; Geschwind, G.; Zhang, K.; Jiang, B.; Mukherjee, D.; Hossain, L.; Piasecki, A. F.; Kabius, B.; Robinson, J. A.; Alem, N. ACS Nano 2015.

(35) Perdew, J. P.; Burke, K.; Ernzerhof, M. Phys. Rev. Lett. 1996, 77 (18), 3865-3868.

(36) Perdew, J. P.; Burke, K.; Ernzerhof, M. Phys. Rev. Lett. 1997, 78 (7), 1396.

(37) Vanderbilt, D. Phys. Rev. B 1990, 41 (11), 7892-7895.

(38) Kresse, G.; Hafner, J. J. Phys. Condens. Matter 1994, 6 (40), 8245-8257.

(39) Kresse, G.; Furthmüller, J. Phys. Rev. B 1996, 54 (16), 11169-11186.

(40) Schweiger, H.; Raybaud, P.; Kresse, G.; Toulhoat, H. J. Catal. 2oo2, 207 (1), 76-87.

(41) Dumcenco, D.; Ovchinnikov, D.; Marinov, K.; Lazić, P.; Gibertini, M.; Marzari, N.; Sanchez, O. L.; Kung, Y.-C.; Krasnozhon, D.; Chen, M.-W.; Bertolazzi, S.; Gillet, P.; Fontcuberta i Morral, A.; Radenovic, A.; Kis, A. ACS Nano 2015.

(42) Penn, R. L.; Banfield, J. F. Science 1998, 281 (5379), 969-971.

(43) Weatherup, R. S.; Bayer, B. C.; Blume, R.; Ducati, C.; Baehtz, C.; Schlögl, R.; Hofmann, S. Nano Lett. 2011, 11 (10), 4154-4160.

(44) Yan, Z.; Lin, J.; Peng, Z.; Sun, Z.; Zhu, Y.; Li, L.; Xiang, C.; Samuel, E. L.; Kittrell, C.; Tour, J. M. ACS Nano 2012, 6 (10), 9110-9117.

(45) Geng, D.; Luo, B.; Xu, J.; Guo, Y.; Wu, B.; Hu, W.; Liu, Y.; Yu, G. Adv. Funct. Mater. 2014, 24 (12), 1664-1670.

(46) Liu, H.; Jiao, L.; Yang, F.; Cai, Y.; Wu, X.; Ho, W.; Gao, C.; Jia, J.; Wang, N.; Fan, H.; Yao, W.; Xie, M. Phys. Rev. Lett. 2014, 113 (6), 066105.

(47) Yin, J.; Yu, J.; Li, X.; Li, J.; Zhou, J.; Zhang, Z.; Guo, W. Small 2015, n/a - n/a.

(48) Karma, A.; Rappel, W.-J. Phys. Rev. E 1998, 57 (4), 4323-4349.

(49) Karma, A.; Plapp, M. Phys. Rev. Lett. 1998, 81 (20), 4444-4447.

(50) Krug, J. In Multiscale Modeling in Epitaxial Growth; Voigt, A., Ed.; ISNM International Series of Numerical Mathematics; Birkhäuser Basel, 2005; pp 69-95.

(51) Meca, E.; Lowengrub, J.; Kim, H.; Mattevi, C.; Shenoy, V. B. Nano Lett. 2013, 13 (11), 5692-5697. 